\section{Porcine Reproductive and Respiratory Syndrome Virus, Thailand, 2010-2011}

\author{
Dachrit Nilubol, Thitima Tripipat, \\ Tawatchai Hoonsuwan, \\ and Khampee Kortheerakul
}

Characterization of porcine reproductive and respiratory syndrome virus (PRRSV) isolates from pigs in Thailand showed 30-aa discontinuous deletions in nonstructural protein 2, identical to sequences for highly pathogenic PRRSV. The novel virus is genetically related to PRRSV from China and may have spread to Thailand through illegal transport of infectious animals from bordering countries.

$\mathrm{P}$ orcine reproductive and respiratory syndrome (PRRS) has a substantial economic effect on the swine industry worldwide. PRRS virus (PRRSV), a member of the family Arteriviridae, is the etiologic agent of the syndrome. PRRSVs are divided into 2 distinct genotypes: type 1 and type 2 . The genotypes have a similar genomic organization, and 10 open reading frames (ORFs) have been identified (1-3). Nonstructural protein 2 (Nsp2) and ORF5 are the most variable regions $(4,5)$, coding for replicase protein and neutralizing epitope, respectively.

In general, PRRSV causes a disease characterized by reproductive failure in sows and respiratory infection in growing pigs. However, in June 2006, a disease characterized by high fever and associated with a high mortality rate emerged in the People's Republic of China (PRC), resulting in the death of $>20$ million pigs (6). The disease, referred to as porcine high fever disease (PHFD), was caused by a new PRRSV variant with a unique hallmark: 2 discontinuous 30-aa deletions in Nsp2. The variant, identified as a highly pathogenic (HP) PRRSV, has subsequently become endemic in PRC (7), and it has spread to other countries, including Vietnam (8) and Lao People's Democratic Republic (Lao PDR) (9).

It is thought that HP-PRRSV spread to Thailand early in 2010. Pigs on a small farm in Nong Khai, a border province in northeastern Thailand located near Lao PDR, showed signs of illness identical to those for PHFD. Within 2 weeks of the initial outbreak, similar clinical features

Author affiliation: Chulalongkorn University, Bangkok, Thailand

DOI: http://dx.doi.org/10.3201/eid1811.111105 were observed in pigs on 19 small farms in a nearby village. Since then, pigs exhibiting similar clinical signs have been observed in $>100$ herds in $>20$ provinces throughout Thailand. The causative agent was isolated from sick pigs and determined to be PRRSV.

To further our knowledge about PRRSV in Thailand, we genetically characterized partial Nsp2 and complete ORF5 genes of PRRSV isolates. In addition, we determined sickness and mortality rates on affected farms.

\section{The Study}

During August 2010-June 2011, outbreaks of disease consistent with PHFD were investigated on 4 pig farms located in geographically separate regions of Thailand (Table 1). Herds were selected for study if farm owners agreed to participate. Pigs in all 4 herds had similar clinical signs. In 3 herds, the outbreak was initially observed in the breeding herd and lasted for $\approx 1$ month; most deaths occurred in the third week. In those 3 herds, the initial signs of illness in sows were inappetence and high fever $\left(40^{\circ} \mathrm{C}-42^{\circ} \mathrm{C}\right)$, followed by reddened skin and abortion. Illness rates among sows were $100 \%, 50 \%$, and $60 \%$, respectively for the 3 herds. The highest number of deaths among the sows occurred within 1 week of onset of the first symptoms. The percentage of culled sows on the 3 farms was $20.4 \%$, $13.6 \%$, and $6.7 \%$, respectively; abortion rates were $52.8 \%$, $8.4 \%$, and $8.7 \%$, respectively (Table 1). The outbreak in the fourth herd was confined to nursery facilities housing $\approx 4,000$ pigs; nearly all pigs were sick within 1 week, and the mortality rate approached $60 \%$ within 2 weeks.

We performed PCR on serum samples from sick pigs to determine the presence of PRRSV; previously reported primers $(7,10)$ were used to amplify partial Nsp2 and complete ORF5 genes. Products were cloned and sequenced at Bio Basic Inc. (Markham, Ontario, Canada). ClustalW (11) was used to align nucleotide and deduced amino acid sequences; 18 partial Nsp2 and 58 complete ORF5 genes were analyzed (Table 2).

To determine the relationship of PRRSV from herds in Thailand to HP-PRRSV, we compared the partial Nsp2 amino acid sequences corresponding to aa 404-640 of ORF1a from the isolates from Thailand with sequences for HP-PRRSV from PRC and Vietnam and for strain VR2332. PRRSV isolates from Thailand possess 2 discontinuous 30-aa deletions (aa 482 and 534-562) that are identical to those in HP-PRRSV (Figure 1).

To analyze the ORF5 genes of isolates from Thailand, PRC, and Vietnam, we constructed a phylogenetic tree by using the distance-based neighbor-joining method as implemented in MEGA4 (12). Bootstrap analysis was performed with 1,000 replicates. The tree showed the co-existence of HP-PRRSV types 1 and 2 in pigs in Thailand (Figure 2). Type 1 isolates from all 4 examined 
Table 1. Characteristics of pig farms with herds infected by PRRSV, Thailand, 2010-2011*

\begin{tabular}{lcccccc}
\hline Herd ID, geographic & & Herd size, & Used attenuated North & \multicolumn{2}{c}{ No. (\%) sow losses } & No. (\%) sows \\
\cline { 5 - 6 } location in country & Production system & no. sows & American PRRSV vaccine & Died & Culled & that aborted \\
\hline UDT, northeast & Farrow-to-wean & 500 & No & $48(9.6)$ & $102(20.4)$ & $264(52.8)$ \\
UD, north & Farrow-to-wean & 1,500 & NK & ND & ND & ND \\
SCP, west & Farrow-to-finish & 500 & Yes & $153(30.6)$ & $68(13.6)$ & $42(8.4)$ \\
FDT, central & Farrow-to-finish & 1,200 & Yes & $29(2.4)$ & $80(6.7)$ & $104(8.7)$ \\
\hline
\end{tabular}

*PRRSV, porcine reproductive and respiratory syndrome virus; ID, identification; NK, not known; ND, no data available.

tData are for the 4 weeks following the start of the outbreak on each farm.

herds clustered with previously reported clusters $(13,14)$ distinct from type 1 modified live vaccine viruses (Porcillis PRRSV and Amervac PRRS). In contrast, some of the type 2 isolates from affected herds in Thailand had formed a novel cluster distinct from previously reported clusters $(13,14)$. The novel type 2 isolates from Thailand clustered with isolates from PRC and Vietnam that were associated with PHFD. Genetic similarities between the novel type 2 isolates and HP-PRRSV were $97.8 \%-98.5 \%$ and $96.5 \%$ $99.0 \%$ homologous at the nucleotide and amino acid levels, respectively. However, the novel type 2 isolates from
Thailand were more closely related to the $07 \mathrm{QN}$ isolate from Vietnam (98.5\% nt and $99.0 \%$ aa similarities) than to the isolates from PRC.

We further investigated routes by which the virus spread. Before the outbreaks in Thailand, dead pigs were illegally transported from Lao PDR to an illegal slaughterhouse located not far from the farm where the first outbreak occurred, and the owner of the farm often visited the slaughterhouse. These findings suggest the movement of infected pigs in neighboring countries might play a role in introducing HP-PRRSV to new regions.
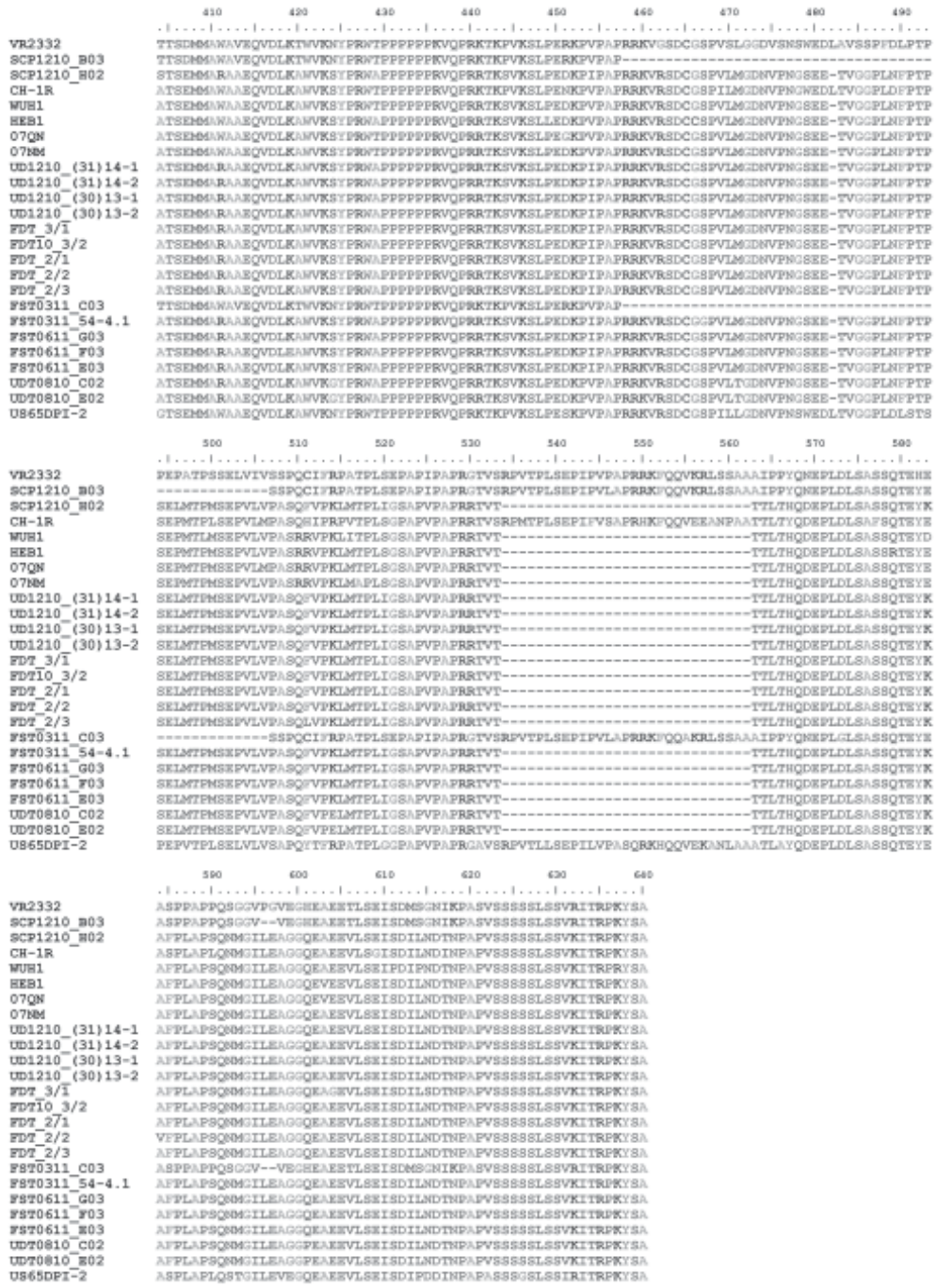

Figure 2. Phylogenetic analysis of types 1 and 2 porcine reproductive and respiratory syndrome virus (PRRSV) isolates constructed by the neighbor-joining method and based on the nucleotide sequences of complete ORF5 genes. The analysis included the following: previous and recent isolates (solid circles) from herds in Thailand that had an outbreak of HPPRRSV; European reference isolates, including Lelystad virus (solid triangle) and 2 type 1 modified live vaccines (Porcilis PRRS, MSD Animal Health, Boxmeer, the Netherlands; and AMERVAC PRRS, Hipra, Girona, Spain) from Europe (open triangles); North American reference isolates, including VR2332 (solid diamond) and North American modified live vaccines (Ingelvac PRRS MLV, Boehringer Ingelheim, St Joseph, MO, USA) (open diamonds); modified live vaccines from PDR (CH1R) (open square); isolates from the People's Republic of China (boldface); and isolate from Vietnam (light gray font). Scale bar indicate nucleotide substitutions per site; numbers at nodes represent the percentage of 1,000 bootstrap replicates. A color version of this figure is available online (wwwnc.cdc. gov/eid/article/18/12/11-1105-F2.htm). 
Infected pigs that were transported across the country and illegal slaughterhouses were the most likely routes of the spread of PRRSV within Thailand. The owners of several of the herds we investigated reported that pigs showed clinical signs within 1-2 days after trucks hauling dead pigs arrived at their farms. It was reported that dead pigs from herds in outbreak areas had been loaded on the trucks the day before they arrived at these farms. In Thailand, unlike in the United States, dead pigs are not composted, buried, or incinerated; instead, they are sold to

\begin{tabular}{|c|c|c|c|c|c|}
\hline Isolate no. & Isolate name & Year and month collected & Genotype & Genes analyzed & GenBank accession no. \\
\hline 1 & UD1210EU24/3 & 2010 Dec & I & ORF5 & JX183110 \\
\hline 2 & UD1210EU23/2 & 2010 Dec & I & ORF5 & JX183111 \\
\hline 4 & SCP1210EU7/79-A07 & 2010 Dec & I & ORF5 & JX183113 \\
\hline 5 & UD1210EU24/2 & $2010 \mathrm{Dec}$ & I & ORF5 & JX183114 \\
\hline 6 & UD1210EU24/1 & 2010 Dec & I & ORF5 & JX183115 \\
\hline 7 & UD1210EU25/2 & $2010 \mathrm{Dec}$ & I & ORF5 & JX183116 \\
\hline 8 & UD1210EU25/1 & 2010 Dec & I & ORF5 & JX183117 \\
\hline 9 & SCP0311EU1/3 & 2011 Mar & I & ORF5 & JX183118 \\
\hline 10 & SCP0311EU1/2 & 2011 Mar & I & ORF5 & JX183119 \\
\hline 11 & FDT0111EU2/3 & 2011 Mar & I & ORF5 & JX183120 \\
\hline 12 & FDT0111EU2/2 & 2011 Mar & I & ORF5 & JX183121 \\
\hline 13 & UD1210EU23/3 & $2010 \mathrm{Dec}$ & I & ORF5 & JX183122 \\
\hline 14 & SCP0311EU1/1 & 2011 Mar & I & ORF5 & JX183123 \\
\hline 15 & FDT0111EU1/2 & 2011 Mar & I & ORF5 & JX183124 \\
\hline 17 & FDT0111EU1/1 & 2011 Mar & I & ORF5 & JX183126 \\
\hline 18 & FDT0111EU2/1 & 2011 Mar & I & ORF5 & JX183127 \\
\hline 19 & SCP0311EU3/2 & 2011 Mar & I & ORF5 & JX183128 \\
\hline 20 & UDT0810US_5/28-160 & $2010 \mathrm{Dec}$ & II & ORF5 & JN255819 \\
\hline 21 & UDT0810US_5/28-161 & $2010 \mathrm{Dec}$ & II & ORF5 & JN255820 \\
\hline 22 & UDT0810US_5/28-162 & $2010 \mathrm{Dec}$ & II & ORF5 & JN255821 \\
\hline 23 & UDT0810US_5/28-163 & 2010 Dec & II & ORF5 & JN255822 \\
\hline 24 & UDT0810US_5/28-164 & 2010 Dec & II & ORF5 & JN255823 \\
\hline 25 & UDT0810US_5/28-165 & 2010 Dec & II & ORF5 & JN255824 \\
\hline 26 & UDT0810US_5/28-166 & $2010 \mathrm{Dec}$ & II & ORF5 & JN255825 \\
\hline 27 & UDT0810US_5/28-167 & $2010 \mathrm{Dec}$ & II & ORF5 & JN255826 \\
\hline 28 & UD1210US/61-E03 & 2010 Dec & II & ORF5 & JN255827 \\
\hline 29 & UD1210US/61-F03 & 2010 Dec & II & ORF5 & JN255828 \\
\hline 30 & UD1210US/61-G03 & 2010 Dec & II & ORF5 & JN255829 \\
\hline 31 & UD1210US/62-H03 & 2010 Dec & II & ORF5 & JN255830 \\
\hline 32 & UD1210US/62-A04 & 2010 Dec & II & ORF5 & JN255831 \\
\hline 35 & FDT10US-2-1 & $2010 \mathrm{Dec}$ & II & ORF5 & JN255834 \\
\hline 36 & FDT10US-2-2 & $2010 \mathrm{Dec}$ & II & ORF5 & JN255835 \\
\hline 37 & FDT10US-2-3 & $2010 \mathrm{Dec}$ & II & ORF5 & JN255836 \\
\hline 38 & SCP1210-U.S.-7-79-1 & 2010 Dec & II & ORF5 & JN255837 \\
\hline 39 & SCP1210-U.S.-7-79-2 & 2010 Dec & II & ORF5 & JN255838 \\
\hline 40 & UDT0810_E02 & $2010 \mathrm{Dec}$ & II & Partial Nsp2 & JN255839 \\
\hline 41 & UDT0810_C02 & $2010 \mathrm{Dec}$ & II & Partial Nsp2 & JN255840 \\
\hline 42 & SCP1210_H02 & $2010 \mathrm{Dec}$ & II & Partial Nsp2 & JN255842 \\
\hline 43 & SCP1210_B03 & 2010 Dec & II & Partial Nsp2 & JN255841 \\
\hline 44 & FST0311_C03 & $2010 \mathrm{Dec}$ & II & Partial Nsp2 & JN255843 \\
\hline 45 & 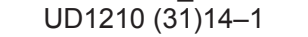 & 2010 Dec & II & Partial Nsp2 & JN255844 \\
\hline 46 & UD1210 (31)14-2 & $2010 \mathrm{Dec}$ & II & Partial Nsp2 & JN255845 \\
\hline 47 & 1-13(30)UD-1 & 2010 Dec & II & Partial Nsp2 & JN255846 \\
\hline 48 & UD1210 (31)13-2 & $2010 \mathrm{Dec}$ & II & Partial Nsp2 & JN255847 \\
\hline 49 & FDT10_3/2 & 2010 Dec & II & Partial Nsp2 & JN255848 \\
\hline 50 & FDT_ $\overline{3} / 1$ & $2010 \mathrm{Dec}$ & II & Partial Nsp2 & JN255849 \\
\hline 51 & FDT_2/1 & $2010 \mathrm{Dec}$ & II & Partial Nsp2 & JN255852 \\
\hline 52 & FDT_2/2 & $2010 \mathrm{Dec}$ & II & Partial Nsp2 & JN255851 \\
\hline 53 & FDT_2/3 & $2010 \mathrm{Dec}$ & II & Partial Nsp2 & JN255850 \\
\hline 54 & FST0311_54-4.1 & $2010 \mathrm{Dec}$ & II & Partial Nsp2 & JN255853 \\
\hline 55 & FST0611_G03 & $2010 \mathrm{Dec}$ & II & Partial Nsp2 & JN255854 \\
\hline 56 & FST0611_F03 & $2010 \mathrm{Dec}$ & II & Partial Nsp2 & JN255855 \\
\hline 57 & FST0611_E03 & $2010 \mathrm{Dec}$ & II & Partial Nsp2 & JN255856 \\
\hline 58 & US65DPI-2 & $2010 \mathrm{Dec}$ & II & Partial Nsp2 & JN255857 \\
\hline
\end{tabular}

${ }^{*}$ PRRSV, porcine reproductive and respiratory syndrome virus; ORF, open reading frame; Nsp2, nonstructural protein 2. 


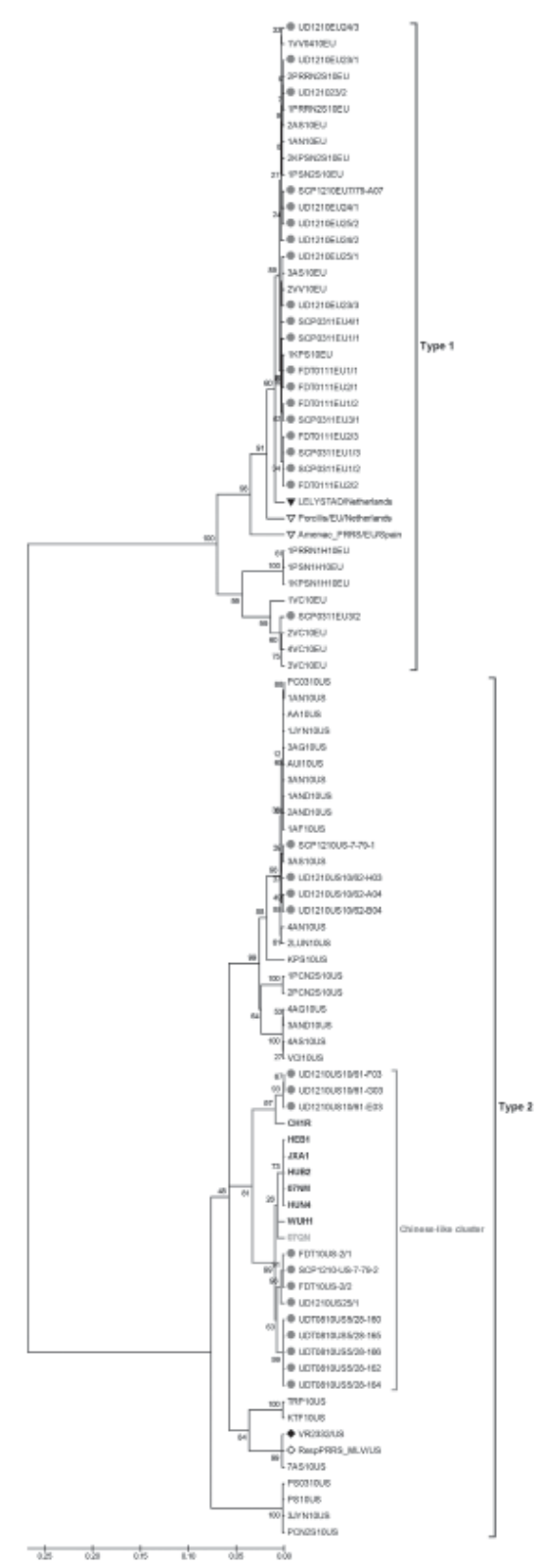

Figure 2. Phylogenetic analysis of types 1 and 2 porcine reproductive and respiratory syndrome virus (PRRSV) isolates constructed by the neighbor-joining method and based on the nucleotide sequences of complete ORF5 genes. The analysis included the following: previous and recent isolates (solid red circles) from herds in Thailand that had an outbreak of HP-PRRSV; European references, including Lelystad virus (solid triangle) and 2 type 1 modified live vaccines (Porcilis PRRS, MSD Animal Health, Boxmeer, the Netherlands; and AMERVAC PRRS, Hipra, Spain) from Europe (open triangles); North American references, including VR2332 (solid diamond) and North American modified live vaccines (Ingelvac PRRS MLV, Boehringer Ingelheim, USA) (open diamonds); modified live vaccines from the People's Republic of China ( $\mathrm{CH} 1 \mathrm{R})$ (open square); isolates from the People's Republic of China (boldface); and isolate from Vietnam (purple font). Scale bar indicate nucleotide substitutions per site; numbers at nodes represent the percentage of 1,000 bootstrap replicates. feed catfish. Truckers associated with this trade visit pig farms to buy and transport dead pigs. These trucks are not washed, so they are a potential source of contamination on farms.

Another source for the introduction of the novel PRRSV into Thailand could be an unapproved vaccine from PRC. The phylogenetic tree demonstrated that 3 recent isolates from Thailand (UD1210US/61-F03, UD1210US/61-G03, and UD1210US/61-E03) were more genetically related to $\mathrm{CH}-1 \mathrm{R}$ (an attenuated vaccine strain used in PRC) than HPPRRSV (Figure 2). CH-1R is a classical PRRSV from PRC that does not possess the 2 discontinuous 30 -aa deletions in Nsp2 (15). Furthermore, CH-1R is an attenuated PRRSV vaccine strain in PRC, and there is evidence that it has been illegally smuggled into Thailand. Thus, it is possible that this modified live virus from PRC may have been administered to the herd involved in the initial outbreak in Thailand and may have been the source of the novel PRRSV strain that caused the outbreak.

\section{Conclusions}

A novel PRRSV, which is genetically related to PRRSV isolates from PRC, has been introduced into Thailand. Sequences of Nsp2 revealed a unique 30-aa discontinuous deletion in the novel virus, a hallmark of HP-PRRSV. The virus may have been introduced into Thailand through the illegal transport of infected materials from bordering countries, more specifically, from Vietnam to Thailand through Lao PDR. This scenario is supported by our finding that PRRSV isolates from Thailand are more homologous with an isolate from Vietnam than with isolates from PRC. The cause of viral spread within Thailand may have been the movement of infected live and dead pigs across the country.

\section{Acknowledgments}

We thank Brad and Eileen Thacker for reviewing the manuscript.

Funds for this research were provided by the Thailand Research Fund (project nos. MRG5080323 and IUG5080001) and by the government of Thailand (budget year 2010).

Dr Nilubol is an assistant professor in the Department of Veterinary Microbiology, Faculty of Veterinary Science, Chulalongkorn University. His research interests are PRRSV and porcine epidemic diarrhea.

\section{References}

1. Meulenberg JJ, Petersen den Besten A, de Kluyver E, van Nieuwstadt A, Wensvoort G, Moormann RJ. Molecular characterization of Lelystad virus. Vet Microbiol. 1997;55:197-202. http://dx.doi. org/10.1016/S0378-1135(96)01335-1 
2. Wu WH, Fang Y, Farwell R, Steffen-Bien M, Rowland RR, Christopher-Hennings J, et al. A 10-kDa structural protein of porcine reproductive and respiratory syndrome virus encoded by ORF2b. Virology. 2001;287:183-91. http://dx.doi.org/10.1006/viro.2001.1034

3. Johnson CR, Griggs TF, Gnanandarajah J, Murtaugh MP. Novel structural protein in porcine reproductive and respiratory syndrome virus encoded by an alternative ORF5 present in all arteriviruses. J Gen Virol. 2011;92:1107-16. http://dx.doi.org/10.1099/ vir.0.030213-0

4. Murtaugh MP, Faaberg KS, Laber J, Elam M, Kapur V. Genetic variation in the PRRS virus. Adv Exp Med Biol. 1998;440:787-94. http://dx.doi.org/10.1007/978-1-4615-5331-1_102

5. Allende R, Lewis TL, Lu Z, Rock DL, Kutish GF, Ali A, et al. North American and European porcine reproductive and respiratory syndrome viruses differ in non-structural protein coding regions. J Gen Virol. 1999;80:307-15.

6. Tian K, Yu X, Zhao T, Feng Y, Cao Z, Wang C, et al. Emergence of fatal PRRSV variants: unparalleled outbreaks of atypical PRRS in China and molecular dissection of the unique hallmark. PLoS ONE. 2007;2:e526. http://dx.doi.org/10.1371/journal.pone.0000526

7. Hu H, Li X, Zhang Z, Shuai J, Chen N, Liu G, et al. Porcine reproductive and respiratory syndrome viruses predominant in southeastern China from 2004 to 2007 were from a common source and underwent further divergence. Arch Virol. 2009;154:391-8. http:// dx.doi.org/10.1007/s00705-009-0316-x

8. Feng Y, Zhao T, Nguyen T, Inui K, Ma Y, Nguyen TH, et al. Porcine respiratory and reproductive syndrome virus variants, Vietnam and China, 2007. Emerg Infect Dis. 2008;14:1774-6. http://dx.doi. org/10.3201/eid1411.071676

9. Ni J, Yang S, Bounlom D, Yu X, Zhou Z, Song J, et al. Emergence and pathogenicity of highly pathogenic porcine reproductive and respiratory syndrome virus in Vientiane, Lao People's Democratic Republic. J Vet Diagn Invest. 2012;24:349-54. http://dx.doi. org/10.1177/1040638711434111
10. Wu J, Li J, Tian F, Ren S, Yu M, Chen J, et al. Genetic variation and pathogenicity of highly virulent porcine reproductive and respiratory syndrome virus emerging in China. Arch Virol. 2009;154:158997. http://dx.doi.org/10.1007/s00705-009-0478-6

11. Thompson JD, Higgins DG, Gibson TJ. CLUSTAL W: improving the sensitivity of progressive multiple sequence alignment through sequence weighting, position-specific gap penalties and weight matrix choice. Nucleic Acids Res. 1994;22:4673-80. http://dx.doi. org/10.1093/nar/22.22.4673

12. Tamura K, Dudley J, Nei M, Kumar S. MEGA4: molecular evolutionary genetics analysis (MEGA) software version 4.0. Mol Biol Evol. 2007;24:1596-9. http://dx.doi.org/10.1093/molbev/msm092

13. Tun HM, Shi M, Wong CL, Ayudhya SN, Amonsin A, Thanawonguwech R, et al. Genetic diversity and multiple introductions of porcine reproductive and respiratory syndrome viruses in Thailand. Virol J. 2011;8:164. http://dx.doi.org/10.1186/1743-422X-8-164

14. Thanawongnuwech R, Amonsin A, Tatsanakit A, Damrongwatanapokin S. Genetics and geographical variation of porcine reproductive and respiratory syndrome virus (PRRSV) in Thailand. Vet Microbiol. 2004;101:9-21. http://dx.doi.org/10.1016/j.vetmic. 2004.03.005

15. Li B, Fang L, Xu Z, Liu S, Gao J, Jiang Y, et al. Recombination in vaccine and circulating strains of porcine reproductive and respiratory syndrome viruses. Emerg Infect Dis. 2009;15:2032-5. http:// dx.doi.org/10.3201/eid1512.090390

Address for correspondence: Dachrit Nilubol, Chulalongkorn University, Veterinary Microbiology, Faculty of Veterinary Science, Henry Dunant Rd, Pathumwan, Bangkok 10230 Thailand; email: dachrit@gmail.com
Get the content you want delivered to your inbox.

Sign up to receive emailed

announcements when new podcasts

or articles on topics you select are

posted on our website.

wwwnc.cdc.gov/eid/subscribe.htm
Table of contents Podcasts Ahead of Print Medscape CME Specialized topics

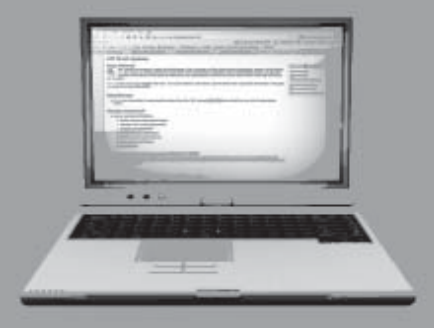

